Saudi Journal of Oral and Dental Research

Abbreviated Key Title: Saudi J Oral Dent Res

ISSN 2518-1300 (Print) |ISSN 2518-1297 (Online)

Scholars Middle East Publishers, Dubai, United Arab Emirates

Journal homepage: https://saudijournals.com/sjodr

Review Article

\title{
Facial Deformity: Cleft Lip and Palate A Recapitulation
}

\author{
Dr. Hadi Yahya Al Bahesh ${ }^{1 *}$, Dr. Hamad Fares Al Munjjim²
}

${ }^{1}$ Najran Specialist, Dental Centre, Najran, Saudi Arabia

${ }^{2}$ Najran, General Hospital, Najran, Saudi Arabia

\author{
DOI: $10.36348 /$ sjodr.2020.v05i06.005 \\ | Received: 07.06.2020 | Accepted: 16.06.2020 | Published: 21.06 .2020 \\ *Corresponding author: Dr. Hadi Yahya Al Bahesh
}

\section{Abstract}

Cleft lip and palate are the utmost typical facial deformity. It may occupy lip only, lip and palate and palate only. The primary deduction of clefting in infants may be each environmental or genetic aspects (such as familial factors and chromosomes). The amount of professionals involves in management of clefting and decides the highest treatment plan depending on the site of deficiency and period of the infant. Aim: The principle of the current paper is to present the etiology of cleft lip and cleft palate mutually the genetic and the environmental aspects. It is optional that the genetic basis for dissimilar kinds of familiar or unusual congenital malformations may marvelously be homogeneous, even as, the ecological basis is heterogeneous. Etiology: The etiology of CLP looks multifaceted, with genetics playing a vital function. Numerous genes create syndromic CLP have been exposed. Three of them, T-box transcription factor-22, poliovirus receptor-like-1, and interferon regulatory factor-6 are accountable for generate X-linked cleft palate, cleft lip/palate-ectodermal dysplasia syndrome, and Vander Woude and popliteal pterygium syndromes, correspondingly; they are also occupied in nonsyndromic CLP. Conclusion: To terminate, while the etiology of nonsyndromic CLP is still mostly unidentified, mutations in applicant genes have been known in a tiny amount of cases. Formative the qualified menace of CLP on the basis of hereditary conditions and environmental manipulate (together with smoking, alcohol use, and dietary factors) will be practical for genetic analysis and the expansion of future protective procedures.

Keywords: Cleft Lip, Cleft Palate, Etiology, Genetic, Multifactorial.

Copyright @ 2020: This is an open-access article distributed under the terms of the Creative Commons Attribution license which permits unrestricted use, distribution, and reproduction in any medium for non-commercial use (NonCommercial, or CC-BY-NC) provided the original author and source are credited.

\section{INTRODUCTION}

A diminutive appraisal of the regular embryonic development of the facial primordia is essential before reviewing the factors that may obstruct with this expansion foremost to clefts of the lip and the palate. In the mounting embryo movement of cell masses, fusion of facial processes and the segregation of tissues are three significant proceedings that guide ultimately to an adult emergence [1].

The shape of development as well as cells react to environmental signals. While both factors are present and interrelate, it is complex to determine the precise function of each of them.

The facial primordia are invented principally of neural crest cells that create from the cranial crest. Neural crest cells travel to the primitive oral cavity wherever, in involvement with ectodermal cells, form the maxillary processes.

Palatal shelves from these processes take place at embryonic day in humans. An intrinsic force, essentially formed by the accretion and hydration of hyaluronic acid-1, is progressively generated within the palatal shelves and reaches a threshold level which exceeds the force of conflict factors.

Fusion and hydration of hyaluronic acid by palatal mesenchyme is encouraged by epidermal growth factor and transforming growth feature beta. The erectile shelf elevating strength is partially intended for by bundles of type I collagen which runs down inside of the vertical shelf from its base to its tip. Furthermore the epithelial covering and connected basement membrane of the palatal shelf display discrepancy traction, which provide to constrain and direct the swelling osmotic energy.

Besides, the palatal mesenchymal cells are themselves contractile and conceal diverse neurotransmitters that influence both mesenchymal cell contractility and glycosaminoglycan dehydration and consequently cooperate a function in palate morphogenesis [7]. 
Epidemiology

Cleft lip with or without palate (CL/P) has a standard birth commonness of 1:700 ranging from 1:500 to $1: 2000$, depending on the contest. There are wide ethnic variations with highest occurrence in Native Americans (3.6:1000), followed by Asians (2.1:1000 Japanese births and 1.7:1000 Chinese births), Caucasians (1:1000), and lowest in those of African decline (0.3:1000).

Cleft palate, which diverge hereditarily from $\mathrm{CL} / \mathrm{P}$, has birth occurrence rate of $1: 2000$ and is additional similar diagonally all populations. About half of the oral clefts occupy lip and palate $(46 \%)$, a third of the clefts involve only the palate $(33 \%)$, and clefts of lip alone account for $21 \%$.

The independent defects arise extra repeatedly on the left side than the right side. Clefts of lip happen in the ratio of 6:3:1 for unilateral left, unilateral right, and mutual. CP is further familiar in females and added frequently linked with additional developmental anomalies. Clefts are indicated to as non-syndromic and syndromic, derived from their connection with extra anomalies.

About $49 \%$ of $\mathrm{CP}$ and $20 \%$ of $\mathrm{CL} / \mathrm{P}$ are connected through a syndrome. Several ordinary syndromes linked with cleft lip and palate comprises Vander Woude, Treacher Collins syndrome, Down syndrome, oro-facial digital syndrome, Opitz syndrome, craniofacial microsomia, and fetal alcohol syndrome $[2$, $3,7]$.

Virtually half of the syndromic cleft palate presentations are interconnected with the harmony of micrognthia, glossoptosis, and airway obstruction (Pierre Robin sequence).

\begin{tabular}{l} 
Distribution of oral clefts \\
Cleft lip and palate $46 \%$ \\
Cleft palate only $33 \%$ \\
Cleft lip only $21 \%$ \\
\hline Cleft lip and palate \\
Average birth prevalence $1: 700$ \\
More common in males \\
Unilateral > bilateral \\
Left side > right side \\
Association with other anomalies $10 \%$ \\
\hline Cleft palate only
\end{tabular}

Average birth prevalence 1:2000

More common in females

Association with other anomalies 50-60\%
Analysis

The embryologic expansion of the face appear at 4 weeks after formation commencing the neural crest ectomesenchyme that forms five prominences; the frontonasal process, and paired maxillary and mandibular processes immediate a innermost despair. Through the fifth and sixth weeks of embryonic progress, bilateral maxillary processes consequent from first brachial arch blend with the medial nasal procedure to outline the upper lip, alveolus, and the primary palate.

The lateral nasal enlargement forms the alar structures of the nose. The lower lip and jaw are shaped by the mandibular processes. This method of development of the face is the result of a flow of processes that employ cell explosion, cell differentiation, cell adhesion, and apoptosis. Breakdown or error in some of these cellular processes that produce union of the medial nasal development with the lateral nasal and maxillary method can effect orofacial clefts.

The molecular measures that underlie these cellular processes are beneath the organize of a firm selection of genes that contain fibroblast growth factors, sonic hedgehog, bone morphogenic proteins, and members of the transforming growth factor beta with additional transcription factors. The pattern of the secondary palate begins during the sixth week after formation beginning the two palatal shelves, which expand from the inside portion of the maxillary processes [2-4].

At some stage in the eighth week, these bilateral maxillary palatal shelves after rising to suitable location above the tongue, fuse with each added and the primary palate. An interruption in the mixture of these embryonic components can happen caused by delay in elevation of the palatal shelves from vertical to horizontal, substandard shelf fusion or post fusion crack ensuing in a cleft of the inferior palate.
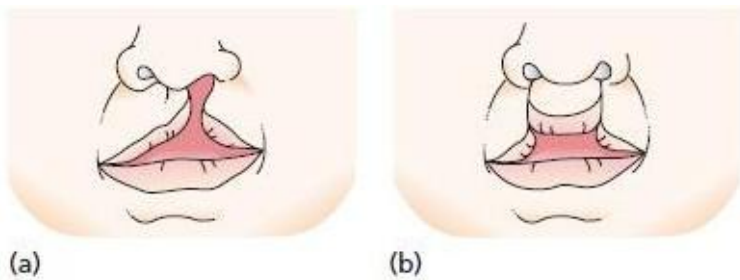

(b)

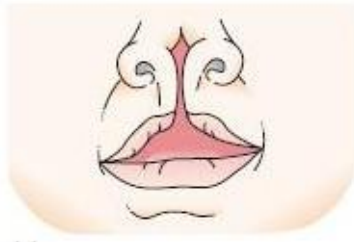

(c)

(d)

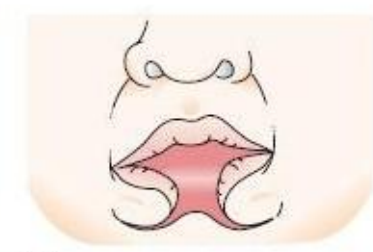

ig-1 


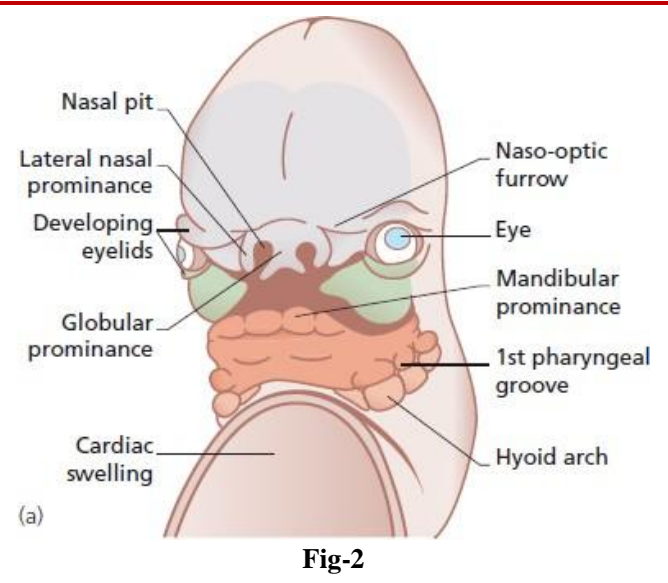

\section{Classification}

At home classify to regulate citations and converse efficiently assorted types of classification systems have been described. The premature Veau's classification integrated groups 1-4 with increasing severity of clefting:

- Group 1 - cleft of the soft palate;

- Group 2 - cleft of the hard and soft palate up to incisive foramen;

- Group 3 - complete unilateral cleft lip and palate;

- Group4-complete bilateral cleft lip and palate.

Even so, this organization is not constantly enough to essay the variations. The additional complicated diagrams, such as the one described by Kernahan and Stark have been used lately. Berkowitz used an uncomplicated classification for labio-palatine clefts:

1. Clefts of lip and alveolus.

2. Clefts of primary (including lip) and secondary palate.

3. Clefts of secondary palate only.

4. Submucous cleft [3].

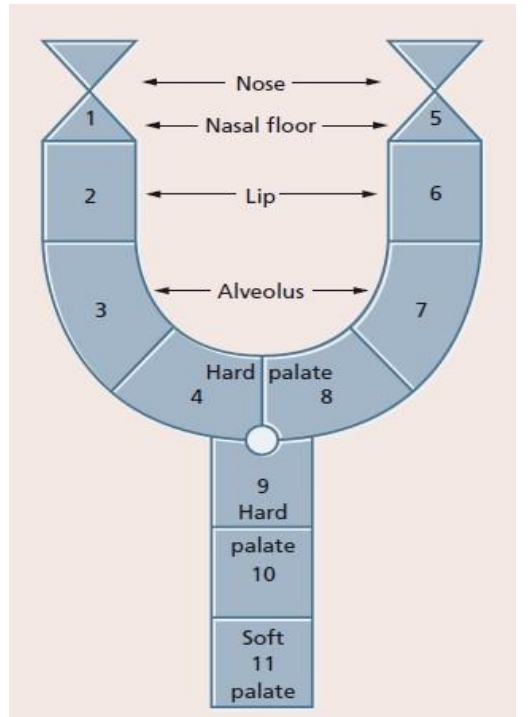

Fig-3: Kernahan and Stark classification of clefts
Universal troubles linked with cleft lip and palate

The cleft lip and palate causes several harms:

\section{Speech Problem}

Patients with a cleft palate have language issues which are effect from velopharyngeal abnormalties. Failure of soft palate to shift upward offer to make contact with nasal cavity consequences in a temporary of air during the nose in place of oral cavity. This situation is well-known as hyper nasality words. Pharyngeal flap and sphincter pharyngoplasty are deliberate as the steadfast operation for correcting the velopharyngeal deficit in patients with a cleft lip and palate.

\section{Hearing Difficulty and Ear Infection}

Otitis media is a form where a liquid is accumulated in the middle ear and consequences in ear illness. This is due to the atypical exploit of Eustachian tube notch by two muscles which are tensor veli palatine and levator veli palatine. This leads to be deficient in of ventilation to the middle ear opening and accretion of solution within the middle ear. This situation is obtainable in the child with cleft palate in the first six months of life [2].

\section{Dental Harms}

Dental problems occupy abnormalities in the dimension and outline of the teeth, Such as, the permanent lateral incisor shows abnormalities in amount and form in the surface of cleft, abnormalities in the location of teeth, interruption of eruption of permanent teeth and impediment of configuration of permanent teeth.

\section{Feeding and Nutritional Troubles}

Feeding problems in children with cleft lip and palate arise because babies are incompetent of sucking either their mother's nipple or from a bottle. Consequently, this affects the burden and enlargement of the baby because the total of milk or food is not sufficient for expansion. There are a assortment of procedure that facilitate the baby to feed and increase a ordinary weight such as the employ of not reusable syringe, spoon and cup and prosthetic obturator device.

\section{Cosmetic Harms}

Patients with cleft lip have cosmetic issues and origin troubles for creation of labial sounds. Babies with cleft lip face complexity when they try to create a contact between upper and lower lips [4].

\section{Psychological Harms}

All exceeding problems damage the psychological surface of a patient with cleft lip and patient where they undergo from dejection, anxiety and lack of esteem and they are incompetent of communicating with their rivals in the school. In addition, various patients experience anxiety because of 
the other people's reactions and concerned regarding assembly people in shared measures.

\section{Treatment}

The purpose of treating a child with a cleft lip and palate are to:

- Present normal speech

- Offer normal feeding

- Advance psychological side

- Present regular dentition and occlusion

- Ordinary hearing

- Complete ordinary aesthetics.

\section{Cleft lip and palate team}

The management involves a squad who consists of a amount of specialists:

- Cleft lip and palate surgeons

- Orthodontist

- Nurse

- Dentist

- Speech and language therapist

- Paediatricians

- Psychologist

- Ear, nose and throat surgeon

- Coordinator manager.

\section{Treatment of Cleft Lip and Palate}

\section{Early Treatment and Evaluation}

Preliminary appraisal of a child with cleft lip is undertaken to discern the degree of the cleft. The parents are learned of the cleft by a specialist nurse. The group frequently discusses with parents the management preparation of their child. Parent feels concern of this deficiency and it is extremely imperative to explain them earlier cases of clefts which were treated to develop the psychological surface. The majority essential thing is the feeding of the infant [3]. A selection of feeding strategy are obtainable and this really on category of clefts. Such as, for infants with secluded cleft lip, a bottle or breast can be used. Alternatively, infants with cleft lip and palate face challenges when feeding they are incompetent of sucking each their mother's nipple or from a bottle. Accordingly, feeding procedure such as nipples, cross curt nipples and longer nipple can be productively supplementary the infant when feeding. The mother can attempt fed her child if she wishes. In case of cleft lip the mother can position her finger over the lip to supply a close between her nipple breast and lip defect. The child should be evaluated hereditarily by an appraisal of the following:

- Prenatal medical history

- $\quad$ several family medical history with clefts

- Assessment of child for congenital heart disease

- Limb and ocular abnormalities

- Every birth abnormalities that may occur with clefts

- Assessment of family members should be undertaken

- To review any genetic factors.

- The child is referred for examination in many departments

- Analysis of cleft both syndromic and non syndromic.

\section{Surgical Treatment \\ Repair of the lip, nose, and anterior palate \\ Patch up of the lip}

This surgical procedure is undertaken regarding three months of child existence and it involves the enlistment of the tissue in the deficiency side of the lip and analysis of orbicularis oris muscle. This permits the conclusion of the lip.

\section{Repair of anterior palate}

Anterior palate restore involves the employ of vomerine flap which is full from nasal septum to seal the deficiency

\section{Repair of the nose}

Nasal repair involves the division the union among ala cartilage and overlying skin and inferior mucosa and then these structures are repositioned once more with diverse methods of obsession.

\section{Alveolar bone grafting}

This surgical treatment involves filling a bony deficiency with cancellous bone and it is performed at the moment of mixed dentition $[1,7]$.

\section{Repair of the Cleft Palate}

Cleft palate surgical procedure is performed concerning 6 month of child life and involves a staffing of mucoperiosteal flaps of the hard palate. Soft palate muscles are then dissected from the bony edge of the hard palate and reposition. An incision is then made in the lateral part of the palate to decline the force in the midline.

\section{Secondary Palate Surgery}

In an assortment of cases palatal repair did not clarify the language problem. Consequently, the surgical treatment is undertaken to present velopharyngeal conclusion among soft palate and pharyngeal wall. The surgical procedure involves the increase of myomucosal flap from the posterior pharyngeal wall and then this flap is attach to the posterior soft palate to realize the conclusion among soft palate and pharyngeal wall [3].

\section{Orthognathic Surgery}

Orthognathic operation is indicated if orthodontic treatment is incapable of establishing an optimum occlusion and aesthetic necessities. This surgical treatment is undertaken to displace the upper and lower jaws in accurate site. In case of maxillary retrognathia, maxillary jaw is repositioned forward with moving the mandible backward to establish class 1 relationship. 


\section{Clinical Psychologists}

Clinical psychologist has an imperative position in the treatment of cleft lip and palate patients. They support to progress the psychological side of the patient in the culture. Children with cleft lip and palate are incapable to communicate with their peers in the school and in society [2].

\section{Possible appliances and their function Nasoalvealar moulding}

Nasoalvealar moulding is presurgical prosthetic prosthesis old in both unilateral and bilateral cleft patients. They proceed to decrease the measure of clefts and assist the conclusion of both lip and palate. In addition they facilitate feeding of the patients because they obdurate the defect. They act to lengthen the columella.

\section{Unilateral cleft lip and palate Fabrication of nasal alveolar moulding}

The construction of NAM devise involves pleasing numerous steps. Primary impression is recorded with silicone putty. The use of alginate and elastomeric materials is contraindicated because they may attach in weaken and cannot be unmoved. The infant is located upturned with supporting head to evade tongue from falling posteriorly. The impression is cast in dental stone and the cleft segments are infertile with wax and then duplicated. Clear acrylic resin is used to fabricate the plate which is fitted to create a normal palate.

\section{Subtractions and additions to NAM}

The clefts either unilateral or bilateral are separated into two segments: greater alveolar and lesser segments. The procedure of moulding involves removing several acrylic resin from various region and calculation to additional areas. This course continues paper until two segments are positioned in seal situation. The intend of calculation and removing acrylic resin is to tolerate the segments to move into reassured areas [1, 3].

\section{Bilaterial cleft lip and palate}

The development of fabrication a nasoalvealar moulding in bilateral clefts is the equivalent as in unilateral cleft lip and palate. The molding of bilateral clefts occupied three stages:

\section{Orthodontic appliance}

To accurate the constricted section and cross bite, numerous appliances such as quad helix and auxiliary appliances are used. This treatment starts after eruption of maxillary incisors (7 year) and first molar tooth. On top to delight malocclusion orthodontic management constantly is accepted out later than eruption of all permanent teeth and this admits the use of fixed appliance brackets are covered on the teeth in organize to shift the teeth into accurate location.

\section{Obturator Devices}

Obturator prostheses are strategy familiar with close the gap among oral and nasal cavities. Two types of cleft lip and palate patients involve the fabrication of obturator devices:

- Patients with hypernasal speech subsequent contravention down of pharyngeal flap.

- Patients with secondary palate.

- Relocate of palatal segments and rotation of premaxilla.

- Move of ala cartilage.

- Elongate the columella.

\section{Clefts with secondary palate}

The method of fabrication of obturator prosthesis for patients with soft palate includes recording master impression; fabrication of acrylic support retained by adam clasps. Artificial teeth are position in case of edentulous and moderately dentate and attempt in meeting is undertaken. Following dispensation the first division of prosthesis, the subsequent part of the prosthesis which covers the soft palate deficiency is attach to the first prosthesis with modeling complex. This prosthesis can be fictitious from of a support which made from metal with addition of a metal loop into center of the soft palate deficiency [5].

\section{Patients with hypernasal speech following Removable Partial Denture}

Several missing teeth in patients with repaired cleft palate can be replaced with removable partial denture. The method of invention of a denture for cleft palate patients is parallel as in non-clefts patients. It involves copy master impression, subsequently fabrication of a cobalt chromium design or, acrylic base, deal of artificial teeth and then dispensation with heat cure acrylic resin [4].

\section{Complete Denture}

The development of invention of a denture for edentulous patients with cleft palate is parallel as in non clefts patients. It involves recording principal impression, production of modified plate followed by copy a master impression poured in dental stone. Fabrication of bite rim copy of a face bow and mounting of a maxillary cast on the articulator are then accomplished. Artificial teeth are approved in the lab and attempt in selection in undertaken for examination the location of the teeth [2].

\section{Osseointegrated Implants}

Implants can be used for preservation of obturator prosthesis in patient with cleft lip and palate together in dentate and edentulous area. Implants are located any in the outstanding maxilla in a superior bone quality or in the zygoma. 


\section{REFERENCES}

1. Shkoukani, M. A., Chen, M., \& Vong, A. (2013). Cleft lip-a comprehensive review. Frontiers in pediatrics, 1(53):1-10.

2. Goodacre, T., \& Swan, M. C. (2008). Cleft lip and palate: current management. Paediatrics and child health, 18(6), 283-292.

3. Muhamad, A. H., Azzaldeen, A., \& Watted, N. (2014). Cleft lip and palate; A comprehensive review. International Journal of Basic and Applied Medical Sciences, 4(1), 338-355.

4. Koch, H., Grzonka, M., \& Koch, J. (1995). Cleft malformation of lip, alveolus, hard and soft palate, and nose (LAHSN) - a critical view of the terminology, the diagnosis and gradation as a basis for documentation and therapy. British Journal of Oral and Maxillofacial Surgery, 33(1), 51-58.

5. Mosahebi, A., \& Kangesu, L. (2006). Cleft lip and palate. Surgery (Oxford), 24(1):33-37.

6. Mossey, P. A., Little, J., Munger, R. G., Dixon, M. J., \& Shaw, W. C. (2009). Cleft lip and palate. The Lancet, 374(9703), 1773-1785.

7. Ysunza, A., Pamplona, M. C., Molina, F., Drucker, M., Felemovicius, J., Ramírez, E., \& Patiño, C. (2004). Surgery for speech in cleft palate patients. International journal of pediatric otorhinolaryngology, 68(12), 1499-1505. 